International Journal of Pure and Applied Mathematics

Volume 106 No. 2 2016, 389-400

ISSN: 1311-8080 (printed version); ISSN: 1314-3395 (on-line version)

url: http://www.ijpam.eu

doi: 10.12732 /ijpam.v106i2.4

ijpam.eu

\title{
THE UPPER VERTEX MONOPHONIC NUMBER OF A GRAPH
}

\author{
P. Titus ${ }^{1 \S}$, K. Iyappan ${ }^{2}$ \\ ${ }^{1}$ Department of Mathematics \\ University College of Engineering Nagercoil \\ Anna University \\ Tirunelveli Region, Nagercoil, 629 004, INDIA \\ ${ }^{2}$ Department of Mathematics \\ V.V. College of Engineering \\ Tirunelveli, 627 657, INDIA
}

\begin{abstract}
For any vertex $x$ in a connected graph $G$ of order $p \geq 2$, a set $S \subseteq V(G)$ is an $x$-monophonic set of $G$ if each vertex $v \in V(G)$ lies on an $x-y$ monophonic path for some element $y$ in $S$. The minimum cardinality of an $x$-monophonic set of $G$ is defined as the $x$-monophonic number of $G$, denoted by $m_{x}(G)$. An $x$-monophonic set $S$ is called a minimal $x$-monophonic set if no proper subset of $S$ is an $x$-monophonic set. The upper $x$-monophonic number, denoted by $m_{x}^{+}(G)$, is defined as the maximum cardinality of a minimal $x$-monophonic set of $G$. We determine bounds for it and find the same for some special classes of graphs. For any two positive integers $a$ and $b$ with $1 \leq a \leq b$, there exists a connected graph $G$ with $m_{x}(G)=a$ and $m_{x}^{+}(G)=b$ for some vertex $x$ in $G$. Also, it is shown that for any three positive integers $a, b$ and $n$ with $a \geq 2$ and $a \leq n \leq b$, there exists a connected graph $G$ with $m_{x}(G)=a, m_{x}^{+}(G)=b$ and a minimal $x$-monophonic set of cardinality $n$.
\end{abstract}

AMS Subject Classification: $05 \mathrm{C} 12$

Key Words: monophonic path, vertex monophonic set, vertex monophonic number, minimal vertex monophonic set, upper vertex monophonic number

\section{Introduction}

By a graph $G=(V, E)$ we mean a finite undirected connected graph without loops or multiple edges. The order and size of $G$ are denoted by $p$ and $q$

Received: October 9, 2015

Published: February 9, 2016

$\S$ Correspondence author
(C) 2016 Academic Publications, Ltd. url: www.acadpubl.eu 
respectively. For basic graph theoretic terminology we refer to Harary [3]. For vertices $x$ and $y$ in a connected graph $G$, the distance $d(x, y)$ is the length of a shortest $x-y$ path in $G$. An $x-y$ path of length $d(x, y)$ is called an $x-y$ geodesic. The neibourhood of a vertex $v$ is the set $N(v)$ consisting of all vertices $u$ which are adjacent with $v$. The closed neibourhood of a vertex $v$ is the set $N[v]=N(v) \bigcup\{v\}$. A vertex $v$ is a simplicial vertex if the subgraph induced by its neighbors is complete. The closed interval $I[x, y]$ consists of all vertices lying on some $x-y$ geodesic of $G$, while for $S \subseteq V, I[S]=\bigcup_{x, y \in S} I[x, y]$. A set $S$ of vertices is a geodetic set if $I[S]=V$, and the minimum cardinality of a geodetic set is the geodetic number $g(G)$. A geodetic set of cardinality $g(G)$ is called a $g$-set. The geodetic number of a graph was introduced in $[1,4]$ and further studied in [2].

The concept of vertex geodomination number was introduced in [5] and further studied in [6]. Let $x$ be a vertex of a connected graph $G$. A set $S$ of vertices of $G$ is an $x$-geodominating set of $G$ if each vertex $v$ of $G$ lies on an $x-y$ geodesic for some element $y$ in $S$. The minimum cardinality of an $x$-geodominating set of $G$ is defined as the $x$-geodomination number of $G$ and is denoted by $g_{x}(G)$. An $x$-geodominating set of cardinality $g_{x}(G)$ is called a $g_{x}-$ set.

A chord of a path $P$ is an edge joining two non-adjacent vertices of $P$. A path $P$ is called a monophonic path if it is a chordless path. For any two vertices $u$ and $v$ in a connected graph $G$, the monophonic distance $d_{m}(u, v)$ from $u$ to $v$ is defined as the length of a longest $u-v$ monophonic path in $G$. The monophonic eccentricity $e_{m}(v)$ of a vertex $v$ in $G$ is $e_{m}(v)=$ $\max \left\{d_{m}(v, u): u \in V(G)\right\}$. A vertex $v$ of $G$ such that $d_{m}(u, v)=e_{m}(u)$ is called a monophonic eccentric vertex of $u$. The monophonic radius, $\operatorname{rad}_{m}(G)$ of $G$ is $\operatorname{rad}_{m}(G)=\min \left\{e_{m}(v): v \in V(G)\right\}$ and the monophonic diameter, $\operatorname{diam}_{m}(G)$ of $G$ is $\operatorname{diam}_{m}(G)=\max \left\{e_{m}(v): v \in V(G)\right\}$. The monophonic distance was introduced and studied in [7].

The concept of vertex monophonic number was introduced by Santhakumaran and Titus in [8]. For a connected graph $G$ of order $p \geq 2$ and a vertex $x$ of $G$, a set $S \subseteq V(G)$ is an x-monophonic set of $G$ if each vertex $v$ of $G$ lies on an $x-y$ monophonic path for some element $y$ in $S$. The minimum cardinality of an $x$-monophonic set of $G$ is defined as the $x$-monophonic number of $G$, denoted by $m_{x}(G)$. An $x$-monophonic set of cardinality $m_{x}(G)$ is called a $m_{x}-$ set of $G$.

These concepts have interesting applications in Channel Assignment Problem in radio technologies. Also, there are useful applications of these concepts 
to security based communication network design. In the case of designing the channel for a communication network, although all the vertices are covered by the network when considering detour monophonic sets, some of the edges may be left out. This drawback is rectified in the case of detour monophonic sets so that considering detour monophonic sets is more advantageous to real life application of communication networks.

The following theorems will be used in the sequel.

Theorem 1. [8] Let $x$ be any vertex of a connected graph $G$. Every simplicial vertex of $G$ other than the vertex $x$ (whether $x$ is simplicial or not) belongs to every $m_{x}-$ set.

Theorem 2. [8] A graph $G$ is complete if and only if $m_{x}(G)=p-1$ for every vertex $x$ in $G$.

Throughout this paper $G$ denotes a connected graph with at least two vertices.

\section{Minimal Vertex Monophonic Sets}

Definition 3. Let $x$ be any vertex of a connected graph $G$. An $x$ monophonic set $S_{x}$ is called a minimal x-monophonic set if no proper subset of $S_{x}$ is an $x$-monophonic set. The upper $x$-monophonic number, denoted by $m_{x}^{+}(G)$, is defined as the maximum cardinality of a minimal x-monophonic set of $G$.

It is clear from the definition that for any vertex $x$ in $G, x$ does not belong to any minimal $x$-monophonic set of $G$.

Example 4. For the graph $G$ given in Figure 2.1, the minimum vertex monophonic sets, the vertex monophonic numbers, the minimal vertex monophonic sets and the upper vertex monophonic numbers are given in Table 2.1. 


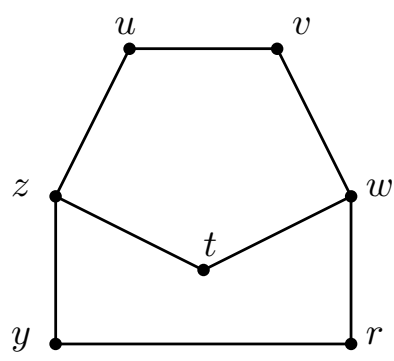

Figure 2.1: $G$

Remark 5. For any vertex $x$ in a connected graph $G$, every minimum $x$-monophonic set is a minimal $x$-monophonic set, but the converse is not true. For the graph $G$ given in Figure 2.1, $\{t, y\}$ is a minimal u-monophonic set but it is not a minimum $u$-monophonic set of $G$.

\begin{tabular}{|c|c|c|c|c|}
\hline Vertex $x$ & Minimum $x$-monophonic sets & $m_{\mathrm{X}}(G)$ & Minimal $x$-monophonic sets & $m_{\mathrm{X}}^{+}(G)$ \\
\hline$u$ & $\{w\},\{r\}$ & 1 & $\{w\},\{r\},\{t, y\}$ & 2 \\
\hline$v$ & $\{z\},\{y\}$ & 1 & $\{z\},\{y\},\{t, r\}$ & 2 \\
\hline$w$ & $\{z\},\{y\},\{u\}$ & 1 & $\{z\},\{y\},\{u\}$ & 1 \\
\hline$r$ & $\{z\},\{u\}$ & 1 & $\{z\},\{u\},\{v, t\}$ & 2 \\
\hline$y$ & $\{v\},\{w\}$ & 1 & $\{v\},\{w\},\{u, t\}$ & 2 \\
\hline$z$ & $\{v\},\{w\},\{r\}$ & 1 & $\{v\},\{w\},\{r\}$ & 1 \\
\hline$t$ & $\{r, u\},\{r, v\},\{y, u\},\{y, v\}$ & 2 & $\{r, u\},\{r, v\},\{y, u\},\{y, v\}$ & 2 \\
\hline
\end{tabular}

Table 2.1

Theorem 6. Let $x$ be any vertex of a connected graph $G$.

(i) Every simplicial vertex of $G$ other than $x$ (whether $x$ is simplicial vertex or not) belongs to every minimal $x$-monophonic set.

(ii) No cut-vertex of $G$ belongs to any minimal $x$-monophonic set.

Proof. (i) Let $x$ be any vertex of $G$. Since $x$ does not belong to any minimal $x$-monophonic set, let $y \neq x$ be a simplicial vertex of $G$. Then $y$ is not an internal vertex of any monophonic path and so $y$ belongs to every minimal $x$ monophonic set of $G$.

(ii) Let $y \neq x$ be a cut-vertex of $G$. Let $U$ and $W$ be two components of $G-\{y\}$. For any vertex $x$ in $G$, let $S_{x}$ be a minimal $x$-monophonic set of $G$. Suppose that $x \in U$. Now, suppose that $S_{x} \bigcap W=\emptyset$. Let $w_{1} \in W$. Then $w_{1} \notin S_{x}$. Since $S_{x}$ is an $x$-monophonic set, there exists an element $z$ in $S_{x}$ such that $w_{1}$ lies in some $x-z$ monophonic path $P: x=z_{0}, z_{1}, \ldots, w_{1}, \ldots, z_{k}=z$ in $G$. Since $S_{x} \bigcap W=\emptyset$ and $y$ is a cut-vertex of $G$, it follows that the $x-w_{1}$ subpath of $P$ and the $w_{1}-z$ subpath of $P$ both contain $y$ so that $P$ is not a path in $G$. Hence $S_{x} \bigcap W \neq \emptyset$. Let $w_{2} \in S_{x} \bigcap W$. Then $w_{2} \neq y$ so that $y$ is an internal vertex of an $x-w_{2}$ monophonic path. If $y \in S_{x}$, let $S=S_{x}-\{y\}$. It 
is clear that every vertex that lies on an $x-y$ monophonic path also lies on an $x-w_{2}$ monophonic path. Hence it follows that $S$ is an $x$-monophonic set of $G$, which is a contradiction to $S_{x}$ a minimal $x$-monophonic set of $G$. Thus $y$ does not belong to any minimal $x$-monophonic set of $G$. Similarly, if $x \in W$, then $y$ does not belong to any minimal $x$-monophonic set of $G$.

Since every end-vertex is a simplicial vertex, the following theorem is an easy consequence of the definition of the upper vertex monophonic number of a graph and Theorem 6 .

Theorem 7. (i) For any non-trivial tree $T$ with $k$ end-vertices, $m_{x}^{+}(T)=$ $k-1$ or $k$ according as $x$ is an end-vertex or not.

(ii) For any vertex $x$ in the complete graph $K_{p}$ of order $p \geq 2, m_{x}^{+}\left(K_{p}\right)=p-1$.

Theorem 8. (i) For any vertex $x$ in the cycle $C_{p}$ of order $p \geq 4$, $m_{x}^{+}\left(C_{p}\right)=1$.

(ii) For the wheel $W_{p}=K_{1}+C_{p-1}(p \geq 5), m_{x}^{+}\left(W_{p}\right)=p-1$ or 1 according as $x$ is $K_{1}$ or $x$ is in $C_{p-1}$.

Proof. (i) Let $C_{p}$ be a cycle. For any vertex $x$ in $C_{p}$, let $y$ be a non-adjacent vertex of $x$. Clearly every vertex of $C_{p}$ lies on an $x-y$ monophonic path and so $\{y\}$ is a minimal $x$-monophonic set of $C_{p}$. Since no adjacent vertex of $x$ lies on a minimal $x$-monophonic set of $C_{p}$, we have $m_{x}^{+}\left(C_{p}\right)=1$.

(ii) Let $x$ be the vertex of $K_{1}$. Clearly $S=V\left(C_{p-1}\right)$ is the unique minimal $x$-monophonic set of $W_{p}$ and so $m_{x}^{+}\left(W_{p}\right)=p-1$.

Let $C_{p-1}: u_{1}, u_{2}, \ldots, u_{p-1}, u_{1}$ be the cycle in $W_{p}$. Let $x$ be any vertex in $C_{p-1}$. Let $y$ be a non-adjacent vertex of $x$ in $W_{p}$. Then any vertex $v$ in $W_{p}$ lies on an $x-y$ monophonic path and so $\{y\}$ is a minimal $x$-monophonic set of $W_{p}$. Since no adjacent vertex of $x$ lies on a minimal $x$-monophonic set of $W_{p}$, we have $m_{x}^{+}\left(W_{p}\right)=1$.

Theorem 9. Let $K_{m, n}(m, n \geq 2)$ be a complete bipartite graph with bipartition $\left(V_{1}, V_{2}\right)$. Then $m_{x}^{+}\left(K_{m, n}\right)= \begin{cases}m-1 & \text { if } x \in V_{1} \\ n-1 & \text { if } x \in V_{2}\end{cases}$

Proof. Let $V_{1}=\left\{u_{1}, u_{2}, \ldots, u_{m}\right\}$ and $V_{2}=\left\{w_{1}, w_{2}, \ldots, w_{n}\right\}$ be a partition of $G$. Let $x \in V_{1}$, say $x=u_{1}$. Since the vertex $u_{i}(2 \leq i \leq m)$ does not lie on any monophonic path starting from $x$ and every vertex of $V_{2}$ lies on an $x-u_{2}$ monophonic path, we have $S_{x}=V_{1}-\{x\}$ is the unique minimal $x$-monophonic set of $G$. Hence $m_{x}^{+}(G)=\left|S_{x}\right|=m-1$. Let $x \in V_{2}$. Then by a similar argument, we get $m_{x}^{+}(G)=n-1$. 
Theorem 10. For $n \geq 2, m_{x}^{+}\left(Q_{n}\right)=1$ for any vertex $x$ in $Q_{n}$.

Proof. Let $x$ be any vertex in $Q_{n}$. Let $y$ be a non-adjacent vertex of $x$ in $Q_{n}$. It is easily seen that every vertex of $Q_{n}$ lies on an $x-y$ monophonic path in $Q_{n}$. Hence $\{y\}$ is a minimal $x$-monophonic set of $G$ and so $m_{x}^{+}(G)=1$.

Theorem 11. Let $G$ be a connected block graph with number of cutvertices $k$. Then $m_{x}^{+}(G)=p-k$ or $p-k-1$ for any vertex $x$ in $G$.

Proof. Let $G$ be a connected block graph. Then every vertex of $G$ is either a cut-vertex or a simplicial vertex and hence by Theorem $6, m_{x}^{+}(G)=p-k$ or $p-k-1$ for any vertex $x$ in $G$.

Theorem 12. For every non-trivial tree $T, m_{x}^{+}(T)=p-d_{m}$ or $p-d_{m}+1$ for any vertex $x$ in $T$ if and only if $T$ is a caterpillar.

Proof. Let $T$ be any non-trivial tree. Let $P: u=v_{0}, v_{1}, \ldots, v_{d_{m}}=v$ be a monophonic diametral path. Let $k$ be the number of end-vertices of $T$ and let $l$ be the number of internal vertices of $T$ other than $v_{1}, v_{2}, \ldots, v_{d_{m}-1}$. Then $d_{m}-1+l+k=p$. By Theorem $7(\mathrm{i}), m_{x}^{+}(T)=k$ or $k-1$ for any vertex $x$ in $T$ and so $m_{x}^{+}(T)=p-d_{m}-l+1$ or $p-d_{m}-l$ for any vertex $x$ in $T$. Hence $m_{x}^{+}(T)=p-d_{m}+1$ or $p-d_{m}$ for any vertex $x$ in $T$ if and only if $l=0$, if and only if all the internal vertices of $T$ lie on the monophonic diametral path $P$, if and only if $T$ is a caterpillar.

\section{Bounds and Realization Results for $m_{x}^{+}(G)$}

Theorem 13. For any vertex $x$ in $G, 1 \leq m_{x}(G) \leq m_{x}^{+}(G) \leq p-1$.

Proof. It is clear from the definition of $x$-monophonic set that $m_{x}(G) \geq$ 1. Since every minimum $x$-monophonic set is a minimal $x$-monophonic set, $m_{x}(G) \leq m_{x}^{+}(G)$. Also, since the vertex $x$ does not belong to any minimal $x$-monophonic set, it follows that $m_{x}^{+}(G) \leq p-1$.

Remark 14. The bounds for $m_{x}(G)$ and $m_{x}^{+}(G)$ in Theorem 13 are sharp. For the cycle $C_{p}(p \geq 4), m_{x}^{+}\left(C_{p}\right)=1$ for any vertex $x$ in $C_{p}$. For the graph $G$ given in Figure 2.1, $m_{t}(G)=m_{t}^{+}(G)=2$. Also, for the complete graph $K_{p}$, $m_{x}^{+}\left(K_{p}\right)=p-1$ for every vertex $x$ in $K_{p}$. All the inequalities in Theorem 13 can be strict. For the graph $G$ given in Figure 2.1, $m_{v}(G)=1, m_{v}^{+}(G)=2$ and $p=7$. Thus $m_{v}(G)<m_{v}^{+}(G)<p-1$. 
Theorem 15. Let $x$ be any vertex in a connected graph $G$ of order $p \geq 3$. If $m_{x}(G)=1$, then $m_{x}^{+}(G) \leq p-2$.

Proof. Let $S_{x}=\{y\}$ be a minimum $x$-monophonic set of $G$ and let $T_{x}$ be a minimal $x$-monophonic set of $G$ with maximum cardinality. Then $y \neq x$. If $y \in T_{x}$, then $T_{x}=S_{x}$ and so $m_{x}^{+}(G)=1 \leq p-2$. If $y \notin T_{x}$, then $m_{x}^{+}(G)=$ $\left|T_{x}\right| \leq p-2$.

Theorem 16. Let $x$ be any vertex in a connected graph $G$. Then $m_{x}(G)=$ $p-1$ if and only if $m_{x}^{+}(G)=p-1$.

Proof. Let $m_{x}(G)=p-1$. Since $m_{x}(G) \leq m_{x}^{+}(G) \leq p-1$, we have $m_{x}^{+}(G)=p-1$. Conversely, let $m_{x}^{+}(G)=p-1$. Then $T=V(G)-\{x\}$ is the minimal $x$-monophonic set of $G$. Now, claim that $m_{x}(G)=p-1$. Otherwise, $G$ has a minimum $x$-monophonic set $T_{1}$ with $\left|T_{1}\right| \leq p-2$. Since $x$ does not belong to any minimum $x$-monophonic set, $T_{1}$ is a proper subset of $T$ and so $T$ is not a minimal $x$-monophonic set of $G$, which is a contradiction.

Theorem 17. For any two integers $a$ and $b$ with $1 \leq a \leq b$, there is $a$ connected graph $G$ with $m_{x}(G)=a$ and $m_{x}^{+}(G)=b$ for some vertex $x$ in $G$.

Proof. We prove this theorem by considering two cases.

Case 1. $1 \leq a=b$. Let $G=K_{a+1}$. Then by Theorems 2 and 7 (ii), $m_{x}(G)=m_{x}^{+}(G)=a$.

Case 2. $1 \leq a<b$. Let $P_{4}: w, x, y, z$ be a path of order 4 . Now, let $G$ be a graph obtained from $P_{4}$ by adding $b$ new vertices $\left\{u_{1}, u_{2}, \ldots, u_{a-1}, v_{1}, v_{2}, \ldots, v_{b-a+1}\right\}$ and joining each $u_{i}(1 \leq i \leq a-1)$ with $x$; and joining each $v_{i}(1 \leq i \leq b-a+1)$ with $w$ and $z$. The graph $G$ is shown in Figure 3.1. Let $S=\left\{u_{1}, u_{2}, \ldots, u_{a-1}\right\}$ be the set of all simplicial vertices of $G$.

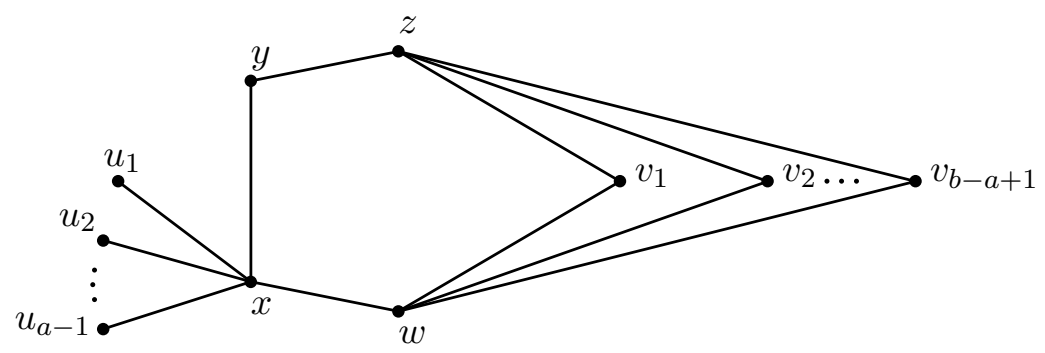

Figure $3.1: G$ 
First, we show that $m_{x}(G)=a$ for the vertex $x$ in $G$. By Theorem 1 , every minimum $x$-monophonic set of $G$ contains $S$. Since $S$ is not an $x$-monophonic set of $G, S_{1}=S \bigcup\{z\}$ is a minimum $x$-monophonic set of $G$ so that $m_{x}(G)=$ $\left|S_{1}\right|=a$.

Next, we show that $m_{x}^{+}(G)=b$. Let $M=\left\{u_{1}, u_{2}, \ldots, u_{a-1}, v_{1}, v_{2}\right.$, $\left.\ldots, v_{b-a+1}\right\}$. It is clear that $M$ is an $x$-monophonic set of $G$. We claim that $M$ is a minimal $x$-monophonic set of $G$. Assume that $M$ is not a minimal $x$-monophonic set of $G$. Then there exists a proper subset $T$ of $M$ such that $T$ is an $x$-monophonic set of $G$. Let $s \in M$ and $s \notin T$. By Theorem 6(i), $s \neq u_{i}$ for all $i=1,2, \ldots, a-1$. Then $s=v_{i}(1 \leq i \leq b-a+1)$. Clearly $v_{i}$ does not lie on any $x-v_{j}$ monophonic path, where $j \neq i$, it follows that $T$ is not an $x$-monophonic set of $G$, which is a contradiction. Thus $M$ is a minimal $x$-monophonic set of $G$ and so $m_{x}^{+}(G) \geq|M|=b$. Also, it is clear that every minimal $x$-monophonic set of $G$ contains at most $b$ elements and hence $m_{x}^{+}(G) \leq b$. Thus $m_{x}^{+}(G)=b$.

Remark 18. The graph $G$ given in Figure 3.1 contains exactly two minimal $x$-monophonic sets, namely $S \bigcup\{z\}$ and $M$. This example shows that there is no "Intermediate Value Theorem" for minimal $x$-monophonic sets, that is, if $n$ is an integer such that $m_{x}(G)<n<m_{x}^{+}(G)$, then there need not exist a minimal $x$-monophonic set of cardinality $n$ in $G$.

Theorem 19. For any three positive integers $a, b$ and $n$ with $a \geq 2$ and $a \leq n \leq b$, there exists a connected graph $G$ with $m_{x}(G)=a, m_{x}^{+}(G)=b$ and a minimal $x$-monophonic set of cardinality $n$.

Proof. Let $P: z_{1}, z_{2}, z_{3}, z_{4}$ and $Q: v_{1}, v_{2}, v_{3}, v_{4}$ be two paths. Let $H$ be the graph obtained from $P$ and $Q$ by identifying the vertices $z_{2}$ in $P$ and $v_{2}$ in $Q$. Let $G$ be the graph obtained from $H$ by adding $b$ new vertices $u_{1}, u_{2}, \ldots, u_{a-2}, y_{1}, y_{2}, \ldots, y_{b-n+1}, x_{1}, x_{2}, \ldots$,

$x_{n-a+1}$ and joining each $u_{i}(1 \leq i \leq a-2)$ with $z_{2}$; joining each $y_{i}(1 \leq i \leq$ $b-n+1)$ with $z_{1}$ and $z_{4}$; and joining each $x_{i}(1 \leq i \leq n-a+1)$ with $v_{1}$ and $v_{4}$ in $H$. The graph $G$ is shown in Figure 3.2.

Let $S=\left\{u_{1}, u_{2}, \ldots, u_{a-2}\right\}$ be the set of all simplicial vertices of $G$ and let $x=z_{2}$. Then by Theorem 1 , every $x$-monophonic set of $G$ contains $S$ and also for any vertex $y \in V(G)-S, S \bigcup\{y\}$ is not an $x$-monophonic set of $G$. It is clear that $S_{1}=S \bigcup\left\{z_{4}, v_{4}\right\}$ is a minimum $x$-monophonic set of $G$ and so $m_{x}(G)=\left|S_{1}\right|=a$. 


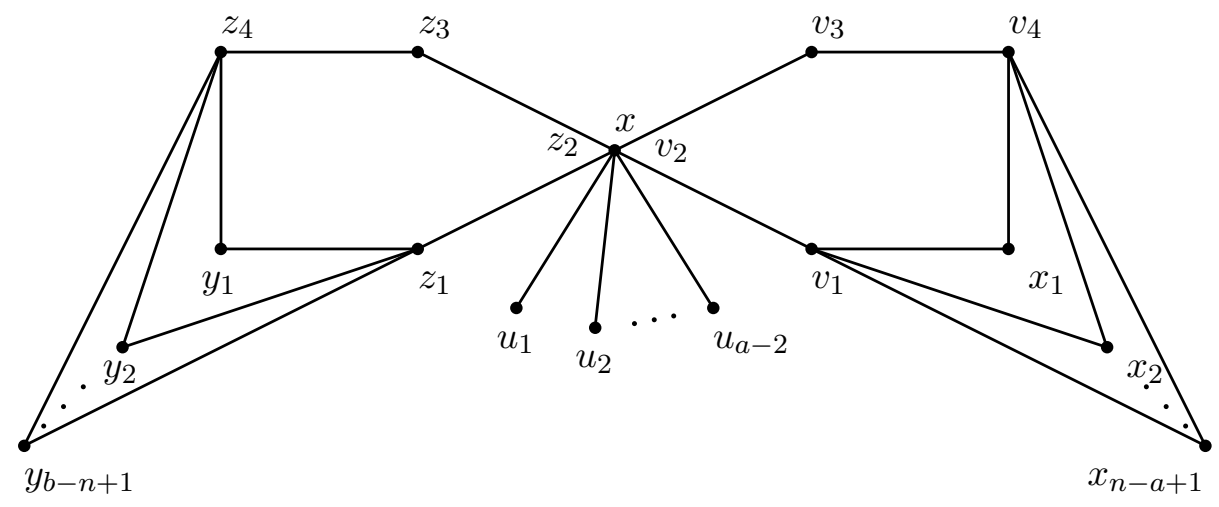

Figure $3.2: G$

Next we show that $m_{x}^{+}(G)=b$. Let $M=S \bigcup\left\{y_{1}, y_{2}, \ldots, y_{b-n+1}\right.$, $\left.x_{1}, x_{2}, \ldots, x_{n-a+1}\right\}$. It is clear that $M$ is an $x$-monophonic set of $G$. We claim that $M$ is a minimal $x$-monophonic set of $G$. Assume that $M$ is not a minimal $x$-monophonic set of $G$. Then there exists a proper subset $M_{1}$ of $M$ such that $M_{1}$ is an $x$-monophonic set of $G$. Let $w \in M$ and $w \notin M_{1}$. By Theorem $6(\mathrm{i})$, either $w=y_{i}(1 \leq i \leq b-n+1)$ or $w=x_{j}(1 \leq j \leq n-a+1)$. If $w=y_{i}(1 \leq i \leq b-n+1)$, then $w$ does not lie on any $x-z$ monophonic path for any $z \in M_{1}$, which is a contradiction. Similarly, if $w=x_{j}(1 \leq j \leq n-a+1)$, then $w$ does not lie on any $x-z$ monophonic path for any $z \in M_{1}$, which is a contradiction. Thus $M$ is a minimal $x$-monophonic set of $G$ and so $m_{x}^{+}(G) \geq|M|=b$. Also, it is clear that every minimal $x$-monophonic set of $G$ contains at most $b$ elements and hence $m_{x}^{+}(G) \leq b$. Hence $m_{x}^{+}(G)=b$.

Finally we show that there is a minimal $x$-monophonic set of cardinality $n$. Let $T=S \bigcup\left\{z_{4}, x_{1}, x_{2}, \ldots, x_{n-a+1}\right\}$. It is clear that $T$ is an $x$-monophonic set of $G$. We claim that $T$ is a minimal $x$-monophonic set of $G$. Assume that $T$ is not a minimal $x$-monophonic set of $G$. Then there is a proper subset $T_{1}$ of $T$ such that $T_{1}$ is an $x$-monophonic set of $G$. Let $t \in T$ and $t \notin T_{1}$. By Theorem 6(i), clearly $t=z_{4}$ or $t=x_{j}(1 \leq j \leq n-a+1)$. If $t=z_{4}$, then $y_{i}(1 \leq i \leq b-n+1)$ does not lie on any $x-y$ monophonic path for some $y \in T_{1}$, which is a contradiction. If $t=x_{j}(1 \leq j \leq n-a+1)$, then $x_{j}$ does not lie on any $x-y$ monophonic path for some $y \in T_{1}$, which is a contradiction. Thus $T$ is a minimal $x$-monophonic set of $G$ with cardinality $n$.

For every connected graph $G, \operatorname{rad}_{m}(G) \leq \operatorname{diam}_{m}(G)$. It is shown in [7] that 
every two positive integers $a$ and $b$ with $a \leq b$ are realizable as the monophonic radius and monophonic diameter, respectively, of some connected graph. It can be extended so that the upper vertex monophonic number can be prescribed.

Theorem 20. For positive integers $r$, $d$ and $n$ with $r \leq d$, there exists a connected graph $G$ with $\operatorname{rad}_{m}(G)=r$, $\operatorname{diam}_{m}(G)=d$ and $m_{x}^{+}(G)=n$ for some vertex $x$ in $G$.

Proof. We prove this theorem by considering three cases.

Case 1. $r=d=1$. Let $G=K_{n+1}$. It is easily seen that $e_{m}(x)=1$ for every vertex $x$ in $G$ and so $\operatorname{rad}_{m}(G)=\operatorname{diam}_{m}(G)=1$. Also, by Theorem $7($ ii), $m_{x}^{+}(G)=n$ for any vertex $x$ in $G$.

Case 2. $1=r<d$. Let $C_{d+2}: v_{1}, v_{2}, \ldots, v_{d+2}, v_{1}$ be a cycle of order $d+2$. Let $G$ be the graph obtained by adding $n-1$ new vertices $u_{1}, u_{2}, \ldots, u_{n-1}$ to $C_{d+2}$ and joining each of the vertices $u_{1}, u_{2}, \ldots, u_{n-1}$ to the vertex $v_{1}$ and also joining each vertex $v_{i}(3 \leq i \leq d+1)$ to the vertex $v_{1}$. The graph $G$ is shown in Figure 3.3. It is easily verified that $1 \leq e_{m}(x) \leq d$ for any vertex $x$ in $G$ and $e_{m}\left(v_{1}\right)=1, e_{m}\left(v_{2}\right)=d$. Then $\operatorname{rad}_{m}(G)=1$ and $\operatorname{diam}_{m}(G)=d$. Let $S=\left\{v_{2}, v_{d+2}, u_{1}, u_{2}, \ldots, u_{n-1}\right\}$ be the set of all simplicial vertices of $G$ and let $x=v_{2}$. Then by Theorem 6(i), every minimal $x$-monophonic set of $G$ contains $S-\left\{v_{2}\right\}$. Clearly $S$ is the unique minimal $x$-monophonic set of $G$ and so $m_{x}^{+}(G)=|S|=n$.

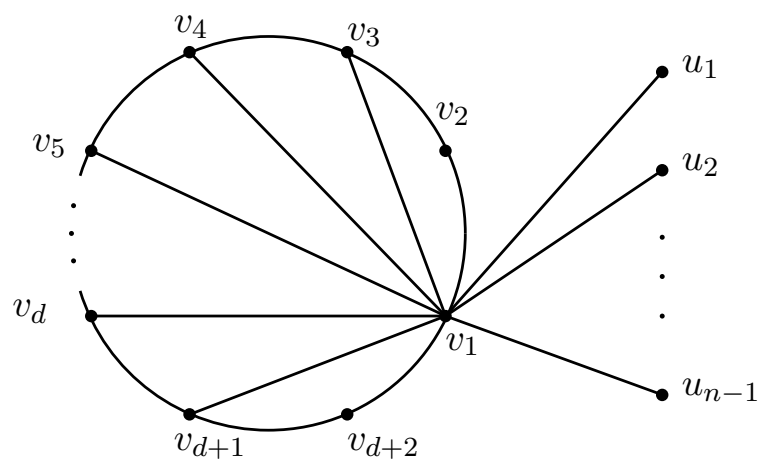

Case 3. $2 \leq r \leq d$. Let $H$ Figure $3.3: G$ abraph obtained from a cycle $C_{r+2}$ : $v_{1}, v_{2}, \ldots, v_{r+2}, v_{1}$ of order $r+2$ and a path $P_{d-r+1}: u_{0}, u_{1}, \ldots, u_{d-r}$ of order $d-r+1$ by identifying the vertex $v_{r+1}$ in $C_{r+2}$ and $u_{0}$ in $P_{d-r+1}$; also join each vertex $u_{i}(1 \leq i \leq d-r)$ in $P_{d-r+1}$ with $v_{r+2}$ in $C_{r+2}$. Now, let $G$ be the graph 
obtained from $H$ by adding $n-1$ new vertices $w_{1}, w_{2}, \ldots, w_{n-1}$ and join each $w_{i}(1 \leq i \leq n-1)$ with $v_{2}$ and $v_{r+2}$ in $H$. The graph $G$ is shown in Figure 3.4.

It is easily verified that $r \leq e_{m}(x) \leq d$ for any vertex $x$ in $G$. Also, $e_{m}\left(v_{r+2}\right)=r$ and $e_{m}\left(v_{1}\right)=d$. It follows that $\operatorname{rad}_{m}(G)=r$ and $\operatorname{diam}_{m}(G)=d$. Now, let $x=u_{d-r}$ and let $S=\left\{v_{1}, w_{1}, w_{2}, \ldots, w_{n-1}\right\}$. Since every vertex of $G$ lies on an $x-y$, where $y \in S$, monophonic path, $S$ is an $x$-monophonic set of $G$. Suppose that $S_{1}$ is a proper subset of $S$ such that $S_{1}$ is an $x$-monophonic set of $G$. Then there exists a vertex $z$ in $S$ such that $z \notin S_{1}$. It is clear that $z$ is either $v_{1}$ or $w_{i}(1 \leq i \leq n-1)$. In all cases $z$ does not lie on any $x-u$, where $u \in S_{1}$, monophonic path, it follows that $S_{1}$ is not an $x$-monophonic set of $G$. This shows that $S$ is a minimal $x$-monophonic set of $G$ and so $m_{x}^{+}(G) \geq n$. Also, it is clear that any minimal $x$-monophonic set of $G$ contains at most $n$ elements and hence $m_{x}^{+}(G) \leq n$. Thus $m_{x}^{+}(G)=n$.

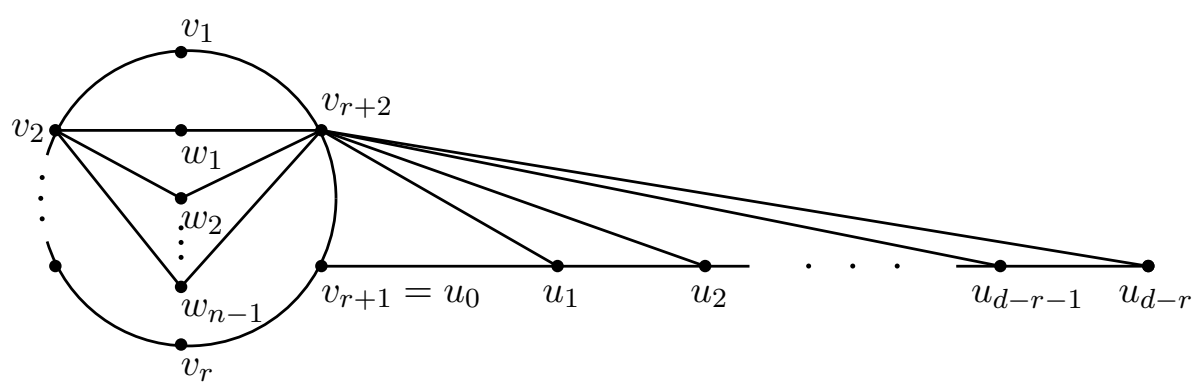

Figure $3.4: G$

\section{References}

[1] F. Buckley, F. Harary, Distance in Graphs, Addison-Wesley, Redwood City, CA, (1990).

[2] G. Chartrand, F. Harary, P. Zhang, On the Geodetic Number of a Graph, Networks, 39 (2002), 1-6.

[3] F. Harary, Graph Theory, Addison-Wesley, (1969).

[4] F. Harary, E. Loukakis, C. Tsouros, The Geodetic Number of a Graph, Math. Comput. Modeling, 17, No. 11 (1993), 87-95.

[5] A.P. Santhakumaran, P. Titus, Vertex Geodomination in Graphs, Bulletin of Kerala Mathematics Association, 2, No. 2 (2005), 45-57.

[6] A.P. Santhakumaran, P. Titus, On the Vertex Geodomination Number of a Graph, Ars Combinatoria, 101 (2011), 137-151.

[7] A.P. Santhakumaran, P. Titus, Monophonic Distance in Graphs, Discrete Mathematics, Algorithms and Applications, 3, No. 2 (2011), 159-169. 
[8] A.P. Santhakumaran, P. Titus, The Vertex Monophonic number of a Graph, Discussiones Mathematicae Graph Theory, 32 (2012), 191-204. 